\title{
Radiolabelling of Mycobacterium avium Oligosaccharide Determinant and Use in Macrophage Studies
}

\author{
By JULIE L. WOODBURY AND WILLIAM W. BARROW* \\ Department of Microbiology and Immunology, Texas College of Osteopathic Medicine, \\ Fort Worth, Texas 76107, USA
}

(Received 21 September 1988; revised 30 January 1989; accepted 22 March 1989)

\begin{abstract}
Internal radiolabelling procedures were used to radiolabel the oligosaccharide determinant of the glycopeptidolipids (GPL) from serovars 4 and 20 of the Mycobacterium avium complex. Mycobacteria were cultured in the presence of $\left[6-{ }^{3} \mathrm{H}\right]$ fucose, $\left[2-{ }^{3} \mathrm{H}\right]$ mannose or [methyl${ }^{3} \mathrm{H}$ ]methionine, after which radiolabelled native lipid was extracted and distribution of radioactivity in native and deacetylated lipid was determined by thin-layer chromatographic methods. Incorporation of radiolabel was confirmed by examining acid hydrolysates of purified GPL for ${ }^{3} \mathrm{H}$-labelled sugars on cellulose thin-layer plates. Least incorporation of radiolabel into GPL was observed with $\left[6-{ }^{3} \mathrm{H}\right] \mathrm{fucose}$, whereas better incorporation was obtained with [2$\left.{ }^{3} \mathrm{H}\right]$ mannose and $\left[\right.$ methyl $\left.{ }^{3} \mathrm{H}\right]$ methionine. Use of $\left[\right.$ methyl $\left.{ }^{3} \mathrm{H}\right]$ methionine resulted in the radiolabelling of the methylated sugars in both the oligosaccharide determinant and the 3,4-di$O$-methylrhamnose located at the terminus of the peptide core. Use of $\left[2-{ }^{3} \mathrm{H}\right]$ mannose resulted in the incorporation of radioactivity into the oligosaccharide determinant as $2-O$-methylfucose, found in the GPL of both serovars 4 and 20 . GPL radiolabelled with $\left[2-{ }^{3} \mathrm{H}\right]$ mannose were subsequently examined in macrophage cultures and found to be relatively inert to degradation by those phagocytic cells. These results substantiate earlier findings with the GPL of serovar 20 and indicate that these mycobacterial components may play a role in pathogenesis.
\end{abstract}

\section{INTRODUCTION}

Currently, disseminated infections caused by members of the Mycobacterium avium complex are a major consideration in the management of individuals suffering from AIDS (Fainstein $e t$ al., 1982; Greene et al., 1982; Kiehn et al., 1985; Masur, 1982). Disseminated M. avium complex infections are difficult to treat in such patients because of their impaired cellular immunity and also because members of the $M$. avium complex are resistant to most of the antitubercular drugs (Davidson et al., 1981; Rosenzweig, 1976; Yeager \& Raleigh, 1973).

The $M$. avium complex is composed of some 31 individual but related serovars that are differentiated on the basis of an array of closely-related polar glycopeptidolipid (GPL) antigens (Brennan \& Goren, 1979) which allow for identification by serological (Schaefer, 1965) and thinlayer chromatographic (TLC) (Brennan et al., 1978) procedures. The general structure of the polar GPL antigens is depicted as a fatty acyl-peptide-sugar core, which is common to all serovars, and an oligosaccharide determinant, which varies from serovar to serovar (Fig. 1) (Brennan \& Goren, 1979). Individual serovars also contain apolar counterparts (aGPL) of the polar GPL antigens which do not possess the oligosaccharide determinant but instead contain only a single 6-deoxytalose which is linked to the peptide moiety of the fatty acyl-peptide-sugar

\footnotetext{
Abbreviations: Ala, alanine; Fuc, fucose, GPL, glycopeptidolipids; aGPL, apolar glycopeptidolipids; dGPL, deacetylated GPL; Man, mannose; Met, methionine; Phe, phenylalanine; Rha, rhamnose; TLC, thin-layer chromatography; TFA, trifluoroacetic acid.
} 


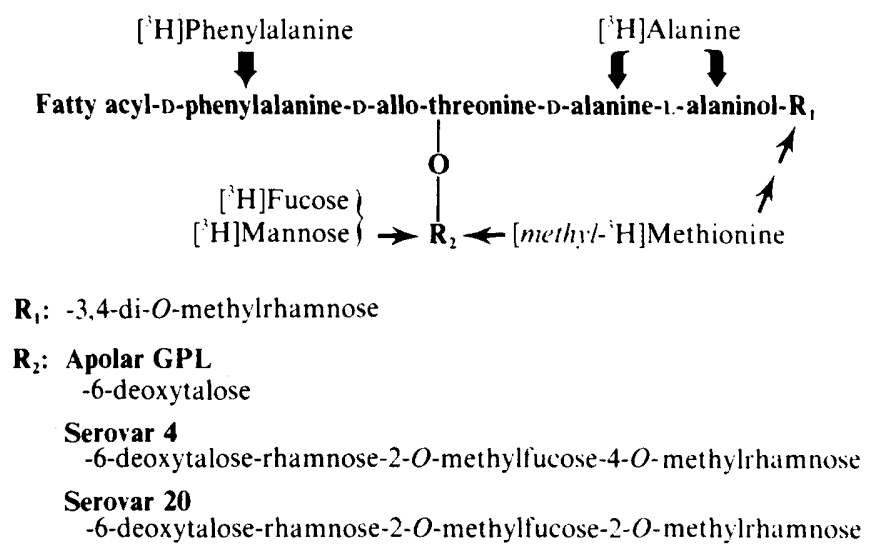

Fig. 1. Structure of apolar GPL components and GPL of serovars 4 and 20, showing incorporation of various radiolabelled components. Incorporation of $\left[{ }^{3} \mathrm{H}\right]$ phenylalanine and $\left[{ }^{3} \mathrm{H}\right]$ alanine was reported in a previous publication (Hooper et al. 1986), and incorporation of $\left[{ }^{3} \mathrm{H}\right]$ fucose, $\left[{ }^{3} \mathrm{H}\right]$ mannose and $[$ methyl$\left.{ }^{3} \mathrm{H}\right]$ methionine are reported in this paper.

core (Fig. 1) (Brennan \& Goren, 1979). The GPL antigens are unique to the $M$. avium complex and represent a major superficial component which is evenly distributed on the surface of the mycobacterial cell (Barrow et al., 1980; Tereletsky \& Barrow, 1983).

Because the GPL antigens represent major constituents of the outermost layer of mycobacteria in the $M$. avium complex, it has been suggested that they play a role in pathogenesis (Draper \& Rees, 1970). For that reason, previous studies in this laboratory have been designed to examine the fate and distribution of the GPL antigens following phagocytosis by host macrophages (Hooper et al., 1986; Tereletsky \& Barrow, 1983). By using radiolabelled GPL antigens, it was determined that the GPL are relatively inert to macrophage degradation (Hooper et al., 1986); however, in that investigation the GPL antigens were radiolabelled with $\left[{ }^{3} \mathrm{H}\right] \mathrm{alanine}$ and $\left[{ }^{3} \mathrm{H}\right]$ phenylalanine, which places the radioactivity in the fatty acyl-peptide moiety that is common to all serovars (Fig. 1). An examination of the postphagocytic handling of the serovar-specific oligosaccharide moiety would be more informative. The purpose of this investigation was to specifically radiolabel the oligosaccharide moiety of the GPL so that the radiolabelled lipids might be used in macrophage degradation studies to examine the fate of the determinant portion of these potentially important mycobacterial components.

\section{METHODS}

Mycobacteria. Mycobacterium avium complex serovar 4 (TMC \# 1463) was obtained from the National Jewish Hospital and Research Center (Denver, Colorado, USA) through Dr Darrel Gwinn, National Institute of Allergy and Infectious Diseases (NIH, Bethesda, Maryland, USA). M. avium serovar 20 has been described previously (Barrow \& Brennan, 1982; Barrow et al., 1980). Mycobacteria were cultivated in 7H9 Middlebrook medium (Difco) supplemented with glycerol and oleic acid-albumin-dextrose (OADC) supplement (Difco) (Brownback \& Barrow, 1988; Hooper et al., 1986) until they reached early exponential phase (100-150 Klett units; KlettSummerson spectrophotometer, no. 42 filter), after which $\left[6-{ }^{-3} \mathrm{H}\right]-\mathrm{L}-$ fucose (specific activity $25 \mathrm{Ci} \mathrm{mmol}^{-1}$, $\left.\left.925 \mathrm{GBq} \mathrm{mmol}^{-1}\right)\left({ }^{3} \mathrm{H}\right] \mathrm{Fuc}\right),\left[2{ }^{3} \mathrm{H}\right]-\mathrm{D}$-mannose (specific activity $\left.30 \mathrm{Ci} \mathrm{mmol}^{-1}, 1110 \mathrm{GBq} \mathrm{mmol}^{-1}\right)\left(\left[{ }^{3} \mathrm{H}\right] \mathrm{Man}\right)$, and $\left[\right.$ methyl $\left.^{-3} \mathrm{H}\right]-\mathrm{L}$-methionine (specific activity $\left.50 \mathrm{Ci} \mathrm{mmol}^{-1}, 1850 \mathrm{GBq} \mathrm{mmol}^{-1}\right)\left({ }^{3} \mathrm{H}\right] \mathrm{Met}$ ) (ICN Radiochemicals) were added individually to cultures at concentrations ranging from 0.25 to $1.0 \mu \mathrm{Ci} \mathrm{ml}^{-1}$. For batch cultures $(1000 \mathrm{ml}),\left[{ }^{3} \mathrm{H}\right]$ Man was added at a concentration of $0.5 \mu \mathrm{Ci} \mathrm{ml}^{-1}$.

Some mycobacterial cultures were cultivated in the presence of $\left[2,3-{ }^{3} \mathrm{H}\right]-\mathrm{L}$-alanine (specific activity $\left.50 \mathrm{Ci} \mathrm{mmol}^{-1}, 1850 \mathrm{GBq} \mathrm{mmol}^{-1}\right)\left(\left[{ }^{3} \mathrm{H}\right] \mathrm{Ala}\right)$ and [side chain- $\left.{ }^{-3} \mathrm{H}\right\}-\mathrm{L}$-phenylalanine (specific activity $25 \mathrm{Ci} \mathrm{mmol}^{-1}$, $\left.\left.925 \mathrm{GBq} \mathrm{mmol}^{-1}\right)\left({ }^{3} \mathrm{H}\right] \mathrm{Phe}\right)(\mathrm{ICN})$ at a concentration of $0.3 \mu \mathrm{Ci} \mathrm{ml}^{-1}$ each (Hooper et al., 1986). Mycobacteria were allowed to grow until they reached stationary phase at 500-550 Klett units, which was equivalent in dry cell weight to $4.4 \mathrm{mg} \mathrm{ml}^{-1}$ (SD, $\pm 0.07, n=4$ ) and $4.5 \mathrm{mg} \mathrm{ml}^{-1}$ ( $\mathrm{sD}, \pm 0.09, n=4$ ) for serovars 4 and 20 , respectively. Cells were then autoclaved, harvested by centrifugation and lyophilized (Hooper $e t$ al., 1986). Lyophilized cells were stored at $-20^{\circ} \mathrm{C}$ until use. 
Chemical procedures. Native lipid was extracted from lyophilized cells with chloroform/methanol $(2: 1, \mathrm{v} / \mathrm{v})$ at $50{ }^{\circ} \mathrm{C}$ as described in previous publications (Hooper et al., 1986; Tereletsky \& Barrow, 1983). Total incorporation of radioactivity was determined by measuring the radioactivity of native lipid in EcoLite scintillation fluid (WestChem, San Diego, California, USA). Distribution of radioactivity in radiolabelled lipid was determined by TLC procedures. Briefly, radiolabelled lipids were applied to silica gel TLC plates and developed in either chloroform/methanol/water $(60: 12: 1$, by vol.) (solvent $\mathrm{A})$ or chloroform/methanol $(11: 1, \mathrm{v} / \mathrm{v})$ (solvent $\mathrm{B}$ ). Following development, $1 \mathrm{~cm}$ sections were transferred to scintillation vials and their radioactivity measured in EcoLite scintillation fluid. Location (cm from origin) of GPL was determined by spraying TLC plates with orcinol/sulphuric acid and heating (Barrow et al., 1980; Brennan \& Goren, 1979).

In some procedures, deacetylated GPL (dGPL) were also examined by TLC. To obtain dGPL, native lipid was treated with $0.2 \mathrm{M}$-methanolic $\mathrm{NaOH}$, as described by Brennan \& Goren (1979). Distribution of radioactivity in $\mathrm{dGPL}$ was determined by TLC as described above.

Purification of both native and deacetylated GPL was accomplished by a one-step adsorption chromatographic procedure described previously (Dimitrijevich et al., 1986). For analysis of sugars, purified GPL were hydrolysed in $2 \mathrm{M}$-trifluoroacetic acid (TFA) $\left(0.2 \mathrm{ml}\right.$ per mg lipid) at $100^{\circ} \mathrm{C}$ for $2 \mathrm{~h}$. Fatty acids were extracted with hexane and sugars were separated on cellulose thin-layer plates in butanol/ethanol/water $(32: 8: 40$, by vol.) (solvent $\mathrm{C}$ ) and detected with aniline/oxalate spray (Horrocks \& Manning, 1949). Distribution of radioactivity was determined by measuring the radioactivity of $1 \mathrm{~cm}$ sections in EcoLite scintillation fluid and identification of sugars was accomplished by using known standards originating from GPL antigens of serovar 20 (Hooper et al., 1986).

Animals. Female and male C57BL/6NHsd mice were purchased from Harlan-Sprague-Dawley, Indianapolis, Indiana, USA, and raised in groups of 10-20 on a diet of mouse chow and water. Mice 5-12 weeks old were used.

Preparation of macrophage monolayers. Resident peritoneal macrophages were obtained from mice by irrigation of the peritoneal cavity with $2.0 \mathrm{ml}$ ice-cold NCTC medium 109 (Difco) containing $5 \mathrm{U}$ heparin (Sigma) per ml of solution, as described in previous publications (Hooper et al., 1986; Tereletsky \& Barrow, 1983). Cell viability was determined by trypan blue exclusion to be $>97 \%$. After dilution to $2 \times 10^{6} \mathrm{cells} \mathrm{ml}^{-1}$, peritoneal cells were added to 24-well tissue culture plates (Falcon Plastics) in $1.0 \mathrm{ml}$ volumes and incubated for $2 \mathrm{~h}$ at $37{ }^{\circ} \mathrm{C}$ under $5 \% \mathrm{CO}_{2}$-inair. Nonadherent cells were removed by washing with medium 109 and plates allowed to incubate overnight to achieve macrophage monolayers as described previously (Hooper et al., 1986).

Addition of radiolabelled GPL to macrophages. Prior to use in macrophage experiments, GPL were maintained in chloroform at $-20^{\circ} \mathrm{C}$. To prepare GPL suspensions, samples were transferred to sterile glass vials and dried under a stream of nitrogen as described previously (Hooper et al., 1986). Medium 109 was added to the dried GPL preparations, followed by sonication in an $80 \mathrm{~W}$ ultrasonic bath (Branson) for 30-60 min at room temperature and refluxing through a 27 gauge needle (Hooper et al., 1986). Each monolayer was treated with an appropriate amount of GPL suspended in a total of $1.0 \mathrm{ml}$ medium 109 for $2 \mathrm{~h}$ at $37^{\circ} \mathrm{C}$ under $5 \% \mathrm{CO}_{2}$-in-air. Following treatment, adherent cells and spent media were assayed for distribution of radioactivity in EcoLite scintillation fluid as described previously (Hooper et al., 1986).

Distribution of radioactivity in chloroform-extractable material. To examine the fate of GPL in macrophage cultures, adherent cell monolayers were treated with $200 \mu \mathrm{g} \mathrm{GPL}$ per $3.2 \times 10^{5}$ cells in $1.0 \mathrm{ml}$ medium 109. At each appropriate time interval, spent medium from three culture wells was removed and added to a $60 \mathrm{ml}$ separating funnel. Macrophages from the respective three wells were lysed and removed by adding $1.0 \mathrm{ml} 0.05 \%$ Triton X-100 (Sigma), as described previously (Hooper et al., 1986). Macrophage lysate was added to the spent medium in the separating funnel and chloroform, methanol and water were added to obtain a solvent ratio of $8: 4: 3$ (by vol.). Mixtures were allowed to stand until two phases had formed, after which the lower chloroform phase was removed, dried under nitrogen, and its radioactivity measured in EcoLite to determine c.p.m. $\mathrm{mg}^{-1}$. The volume of the upper methanol/water phase was measured in a graduated cylinder; $1.0 \mathrm{ml}$ samples were removed and their radioactivity measured in EcoLite to determine the total c.p.m. $\mathrm{ml}^{-1}$.

\section{RESULTS}

\section{Incorporation of radioactivity into native lipid}

Preliminary studies were conducted on a small scale to determine if sufficient incorporation of radiolabel into GPL could be accomplished with $\left[{ }^{3} \mathrm{H}\right] \mathrm{Fuc},\left[{ }^{3} \mathrm{H}\right]$ Man or $\left[{ }^{3} \mathrm{H}\right]$ Met. Mycobacteria were cultured in $100 \mathrm{ml}$ volumes in the presence of each radiolabelled component at concentrations of $0 \cdot 25,0.5$ and $1.0 \mu \mathrm{Ci}$ (ml culture $)^{-1}$. Native lipid was extracted and the amount of radioactivity determined as c.p.m. (mg lipid) $)^{-1}$; the distribution of radioactivity was examined to determine whether the radiolabelled component was appropriate for use in subsequent macrophage studies involving the GPL. 


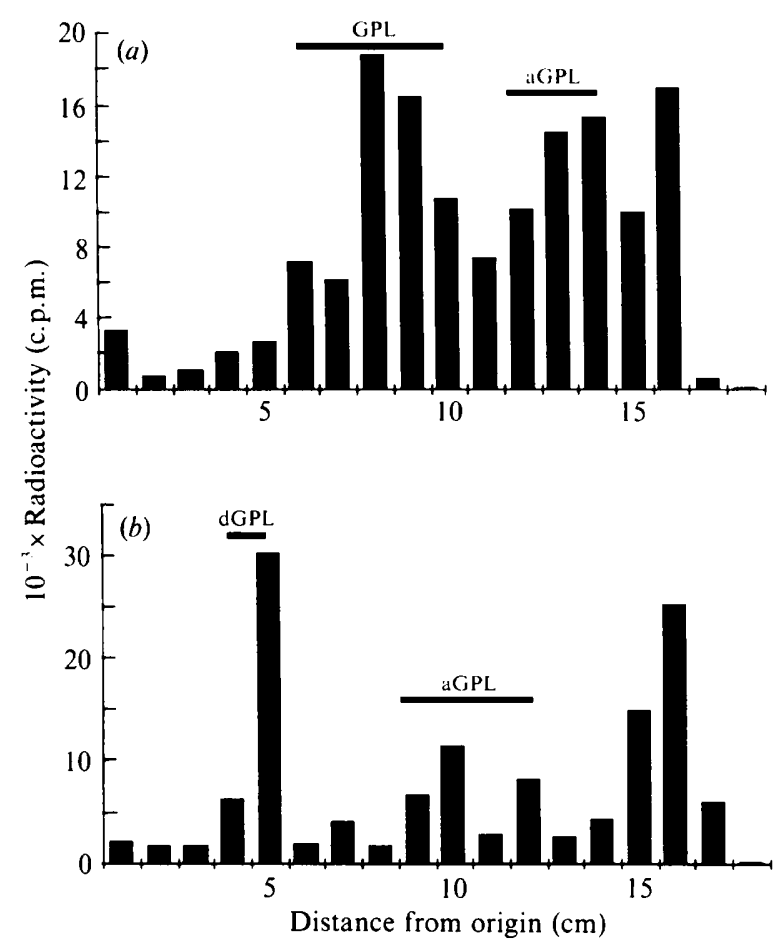

Fig. 2. Distribution on TLC of radioactivity in native lipid $(a)$ and deacetylated lipid $(b)$ from $\left[{ }^{3} \mathrm{H}\right] \mathrm{Met}-$ radiolabelled serovar 4. Plates were developed in solvent A, and GPL, deacetylated GPL (dGPL) and apolar GPL (aGPL) components detected by spraying with orcinol/sulphuric acid. Percentage distribution of radioactivity ( $\pm \mathrm{SD})$ was determined as: $(a) \mathrm{GPL}=40 \%, n=2 ;$ aGPL $=37 \%, n=2$ and $(b)$ dGPL $=22 \% \pm 2 \cdot 6, n=3 ;$ aGPL $=24 \% \pm 2, n=3$. The absolute value corresponding to $100 \%$ was $(a) 1.5 \times 10^{5}$ c.p.m. $n=2$ and $(b) 5.9( \pm 0.9) \times 10^{4}$ c.p.m., $n=3$.

Examination of radiolabelled lipid indicated that use of $\left[{ }^{3} \mathrm{H}\right]$ Fuc resulted in the least incorporation into native lipid. Incorporation of $\left[{ }^{3} \mathrm{H}\right] \mathrm{Fuc}$ into native lipid of serovar 4 amounted to $7.2 \times 10^{3}, 9.0 \times 10^{3}$ and $10.0 \times 10^{3}$ c.p.m. (mg lipid) ${ }^{-1}$ and $1.5 \times 10^{3}, 2.0 \times 10^{3}$ and $2.7 \times 10^{3}$ c.p.m. (mg lipid) $)^{-1}$ for serovar 20 when concentrations of $0.25,0.5$ and $1.0 \mu \mathrm{Ci} \mathrm{ml}^{-1}$ were used. Examination of the distribution of radioactivity in the $\left[{ }^{3} \mathrm{H}\right]$ Fuc-labelled lipid from serovars 4 and 20 by TLC revealed that only a minimal amount was incorporated into the GPL. Because of the low incorporation of radioactivity into native lipid, $\left[{ }^{3} \mathrm{H}\right] \mathrm{Fuc}$ was not considered for further use as a probe in subsequent macrophage uptake studies involving radiolabelled GPL.

Using $\left[{ }^{3} \mathrm{H}\right] \mathrm{Man}$, the mean incorporation into native lipid from serovar 4 was $10 \cdot 4 \times 10^{3}, 17 \cdot 3$ $\times 10^{3}$ and $20 \times 10^{3}$ c.p.m. (mg lipid) $)^{-1}(n=2)$, and for serovar 20 it was $13 \cdot 1 \times 10^{3}, 28.6 \times 10^{3}$ and $52.4 \times 10^{3}$ c.p.m. (mg lipid) ${ }^{-1}$ (one experiment), for concentrations of $0.25,0.5$ and $1.0 \mu \mathrm{Ci} \mathrm{ml}{ }^{-1}$, respectively. Use of $\left[{ }^{3} \mathrm{H}\right] \mathrm{Met}$ resulted in incorporation into native lipid from serovar 4 of $88.9 \times 10^{3}, 529 \times 10^{3}$ and $1080 \times 10^{3}$ c.p.m. (mg lipid) $)^{-1}$, and for serovar 20 , incorporation of $74.9 \times 10^{3}, 124 \times 10^{3}$ and $230 \times 10^{3}$ c.p.m. (mg lipid) ${ }^{-1}$ (one experiment), for concentrations of $0.25,0.5$ and $1.0 \mu \mathrm{Ci} \mathrm{ml}^{-1}$, respectively.

\section{Distribution of radiolabel in native lipid}

Analysis of serovar 4 [methyl $\left.{ }^{3} \mathrm{H}\right]$ Met-radiolabelled lipid by TLC in solvent A (Fig. $2 a$ ) revealed that radioactivity was distributed over a wide area, with $40 \%(n=2)$ and $37 \%(n=2)$ of the radioactivity localized in the area corresponding to GPL and aGPL, respectively. Examination of deacetylated serovar $4\left[\right.$ methyl $\left.{ }^{-3} \mathrm{H}\right]-$ Met-radiolabelled lipid by TLC in solvent A (Fig. $2 b$ ) confirmed that $22 \%$ (SD, $\pm 2 \cdot 6, n=3$ ) of the radioactivity was localized in an area 

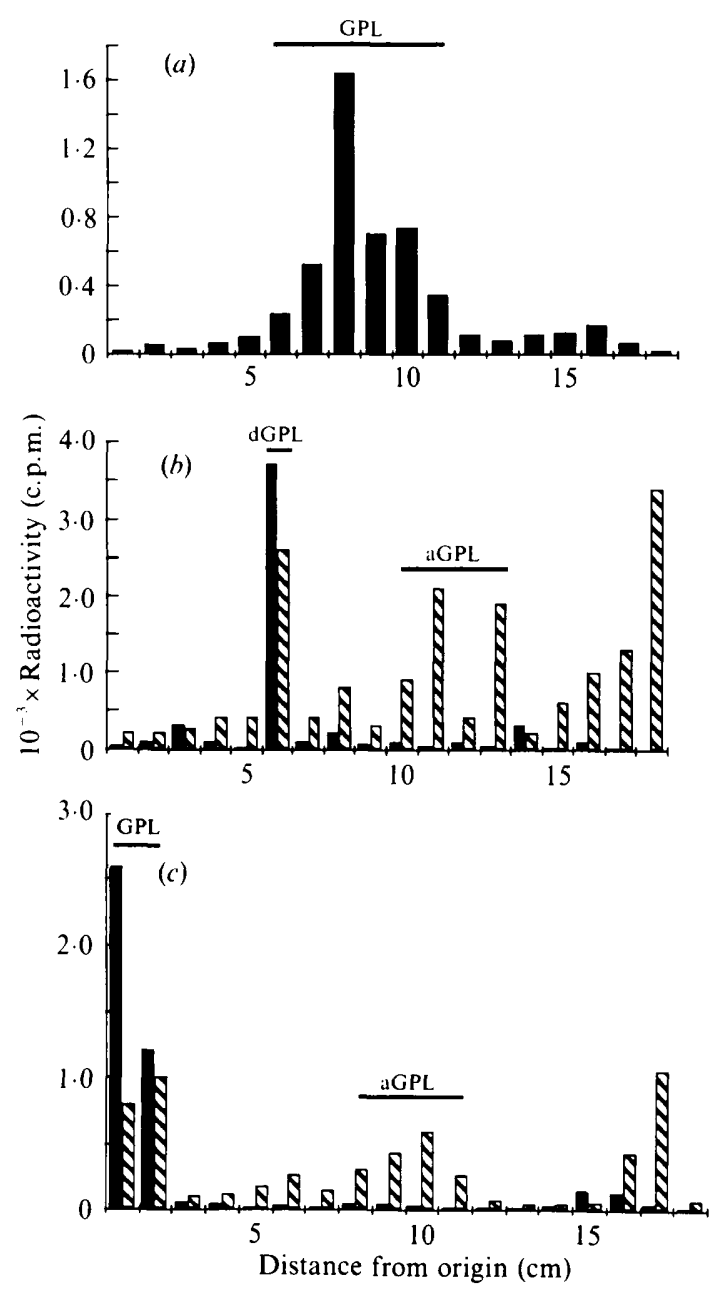

Fig. 3. Distribution on TLC of radioactivity in native lipid $(a, c)$ and deacetylated lipid $(b)$ from $\left[{ }^{3} \mathrm{H}\right]$ Man-radiolabelled $(\square)$ and $\left[{ }^{3} \mathrm{H}\right] \mathrm{Phe} /$ Ala-radiolabelled $(\mathbb{Q})$ serovar 4. Plates were developed in solvent A $(a, b)$ or solvent B (c), and GPL, deacetylated GPL (dGPL) and apolar GPL (aGPL) components detected by spraying with orcinol/sulphuric acid. Percentage distribution of radioactivity ( \pm SD) was determined as: (a) GPL $=57 \% \pm 6 \cdot 7, n=3 ;(b)$ dGPL $=64 \%, n=2(\square)$ and $15 \%, n=2$

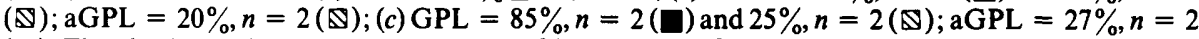
$(\mathbb{\nabla})$. The absolute value corresponding to $100 \%$ was $2.9 \times 10^{3}$ c.p.m., $n=2(\square)$ and $5.2 \times 10^{3}$ c.p.m.,

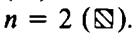

corresponding to only the dGPL and $24 \%(\mathrm{SD}, \pm 2, n=3)$ in an area corresponding to the aGPL components. Examination of the deacetylated lipid is more accurate because the dGPL can be confined to a single $1 \mathrm{~cm}$ section, thus allowing for a more precise assessment of radioactivity distribution. Similar findings were observed for serovar 20 (data not presented) and are consistent with findings reported previously in which it was determined that $\left[\right.$ methyl $\left.{ }^{3} \mathrm{H}\right] \mathrm{Met}$ radiolabelled the methylated sugars of the GPL antigens (Barrow et al., 1980).

Examination of $\left[{ }^{3} \mathrm{H}\right]$ Man-radiolabelled lipid from serovar 4 by TLC in solvent A (Fig. $3 a$ ) revealed that the majority $(57 \% ; \mathrm{SD}, \pm 6 \cdot 7, n=3)$ of the radioactivity was localized in an area corresponding to only the GPL and not the aGPL. Lack of incorporation into the aGPL was established by comparing the TLC distribution of radioactivity from deacetylated $\left[{ }^{3} \mathrm{H}\right] \mathrm{Man}$ radiolabelled lipid with that of deacetylated $\left[{ }^{3} \mathrm{H}\right] \mathrm{Phe} / \mathrm{Ala}$-radiolabelled lipid in solvent $\mathrm{A}$ (Fig. $3 b$ ). In the case of the $\left[{ }^{3} \mathrm{H}\right] \mathrm{Man}$-radiolabelled lipid, radioactivity was localized almost entirely in the area corresponding to the dGPL (mean $64 \% ; n=2$ ) and not the aGPL components (Fig. 
$3 b$ ). Likewise, when native lipid from serovar 4 was developed in solvent $\mathbf{B}$, for better separation of aGPL components, it was further demonstrated that radioactivity was detected in the area corresponding to the aGPL for only lipid radiolabelled with $\left[{ }^{3} \mathrm{H}\right] \mathrm{Phe} / \mathrm{Ala}$ (mean $27 \% ; n=2$ ) and not $\left[{ }^{3} \mathrm{H}\right] \mathrm{Man}$ (Fig. $3 c$ ). Use of $\left[{ }^{3} \mathrm{H}\right] \mathrm{Phe} / \mathrm{Ala}$ has been shown to lead to incorporation of radiolabel into the peptide core which is common to both the GPL and the aGPL (Hooper et al., 1986). Although a certain percentage of radioactivity was observed in the upper region of the plate (i.e. cm 15, Fig. $3 b, c$ ), this was not associated with the aGPL components. The identity of that component is the subject of another investigation.

\section{Purification of native and deacetylated GPL}

Native and deacetylated radiolabelled lipids from serovars 4 and 20 were purified by a onestep chromatographic procedure (Dimitrijevich et al., 1986). Results for purification of GPL antigens from serovar 20 have been reported previously (Dimitrijevich et al., 1986). For serovar 4, lipid was first eluted in chloroform/methanol $(96: 4, \mathrm{v} / \mathrm{v})$ to remove pigments, followed by elution in chloroform/methanol $(93: 7, \mathrm{v} / \mathrm{v})$ to remove the GPL. Yield of pure GPL from serovar 4 averaged $14 \%$ of total lipid.

\section{Distribution of radiolabel in sugars from purified GPL}

To further confirm the incorporation of radiolabel into the GPL, purified deacetylated radiolabelled GPL from serovars 4 and 20 were hydrolysed in 2 M-TFA and hydrolysates examined by development on cellulose TLC. Sugars in the TFA hydrolysates were identified by development on cellulose TLC along with standards obtained from serovar 20 (Barrow et al., 1980). Analysis of the hydrolysate from $\left[\right.$ methyl $\left.-{ }^{3} \mathrm{H}\right] \mathrm{Met}$-radiolabelled GPL of serovar 4 revealed that radioactivity was localized in the area corresponding to $2-O$-methylfucose $(2-O-\mathrm{MeFuc}), 4-$ $O$-methylrhamnose (4-O-MeRha), and 3,4-di- $O$-methylrhamnose (3,4-di- $O$-MeRha) (data not shown). Analysis of sugars in the TFA-hydrolysate of purified $\left[{ }^{3} \mathrm{H}\right] \mathrm{Man}$-labelled serovar $4 \mathrm{GPL}$ revealed that only $2-O-\mathrm{MeFuc}$ was radiolabelled (data not shown). This identical pattern was also observed for GPL antigens from serovar 20, with the exception that 2-O-MeRha replaced 4$O$-MeRha. Therefore, for specific incorporation of radiolabel into the oligosaccharide moiety of the GPL, $\left[{ }^{3} \mathrm{H}\right]$ Man was determined to be the radiolabelled component of choice because of its ability to be incorporated into the 2-O-MeFuc moiety of the oligosaccharide determinant, both for serovar 4 and serovar 20 (Fig. 1).

\section{Uptake of radiolabelled GPL by mouse peritoneal macrophages}

Unelicited peritoneal cell counts from C57BL/6 mice averaged $4.2 \times 10^{6}$ cells $\mathrm{ml}^{-1}$ (sD, $\pm 1.4 \times 10^{6}, n=7$ ). The area of each tissue culture well was $2 \mathrm{~cm}^{2}$, and the addition of $2 \times 10^{6}$ peritoneal cells to each well resulted in an average macrophage monolayer of $3.2 \times 10^{5}$ cells (SD, $\pm 0.7 \times 10^{5}, n=7$ ).

Uptake of GPL by peritoneal macrophages was determined by pulsing adherent cells with varying concentrations of serovar 4 GPL $\left(10-250 \mu \mathrm{g} \mathrm{GPL} \mathrm{ml}^{-1}\right.$ per $3 \cdot 2 \times 10^{5}$ cells $)$. GPL used in the uptake studies were radiolabelled with $\left[{ }^{3} \mathrm{H}\right] \mathrm{Phe} / \mathrm{Ala}$ and their ${ }^{3} \mathrm{H}$ radioactivity was $1.5 \times 10^{5}$ c.p.m. $\mathrm{mg}^{-1}$. The concentration for maximum uptake appeared to be slightly above $250 \mu \mathrm{g} \mathrm{GPL} \mathrm{ml}{ }^{-1}$ (Fig. 4). The mean percentage uptake $( \pm \mathrm{SD})$ of $\left[{ }^{3} \mathrm{H}\right] \mathrm{Phe} /$ Ala-radiolabelled GPL was $17 \pm 1 \cdot 2,19 \pm 2 \cdot 0,19 \pm 1 \cdot 1,19 \pm 1 \cdot 4,19 \pm 0 \cdot 6$ and $20 \pm 1 \cdot 3(n=3)$, for GPL concentrations of $10,20,50,100,200$ and $250 \mu \mathrm{g} \mathrm{ml}^{-1}$, respectively. This is in contrast to the percentage uptake for serovar $20 \mathrm{GPL}$ antigens, which ranged from 60 to $76 \%$ for GPL concentrations of $10-250 \mu \mathrm{g} \mathrm{ml}^{-1}$ (Hooper et al., 1986). To be consistent with previous studies involving GPL antigens from serovar 20 , a concentration of $200 \mu \mathrm{g} \mathrm{GPL} \mathrm{ml}^{-1}$ was chosen as the optimum concentration for macrophage degradation studies to follow.

\section{Distribution of radioactivity in chloroform-extractable material from macrophage cultures}

Degradation of GPL by mouse peritoneal macrophages was examined by using $\left[{ }^{3} \mathrm{H}\right] \mathrm{Man}$ radiolabelled GPL. As compared to the $\left[{ }^{3} \mathrm{H}\right] \mathrm{Phe} / \mathrm{Ala}$-radiolabelled serovar $20 \mathrm{GPL}$ antigens used in our previous study (Hooper et al., 1986), the $\left[{ }^{3} \mathrm{H}\right] \mathrm{Man}$-radiolabelled GPL from serovar 4 


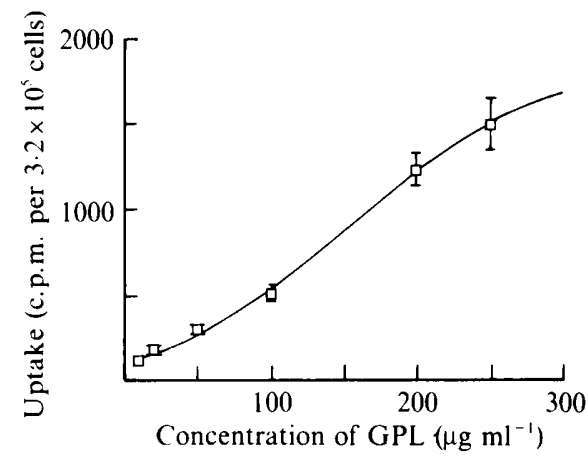

Fig. 4

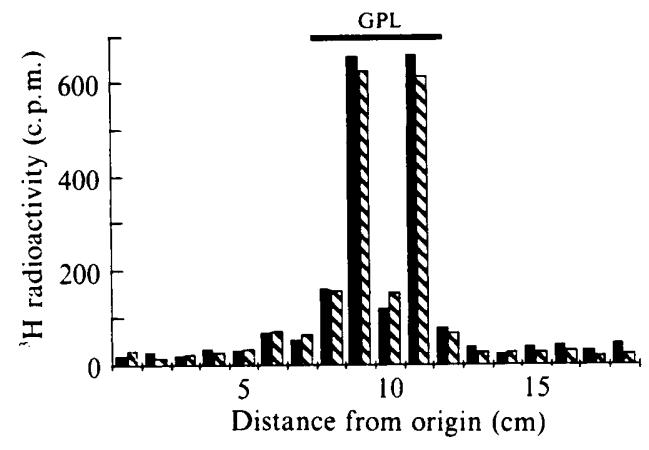

Fig. 5

Fig. 4. Uptake of radiolabelled GPL by mouse peritoneal macrophages. Macrophage monolayers were pulsed for $2 \mathrm{~h}$ with $\left[{ }^{3} \mathrm{H}\right] \mathrm{Phe} /$ Ala-radiolabelled GPL (radioactivity $1.5 \times 10^{5}{ }^{3} \mathrm{H} \mathrm{c.p.m.} \mathrm{mg}^{-1}$ ) from serovar 4. Bars represent SD $(n=3)$.

Fig. 5. Distribution on TLC of chloroform-extractable components from spent media and macrophages obtained from peritoneal macrophage cultures at $0 \mathrm{~h}(\mathrm{Q})$ and $96 \mathrm{~h}(\mathbb{S})$ following pulse with purified $\left[{ }^{3} \mathrm{H}\right]$ Man-radiolabelled serovar 4 GPL. Plates were developed in solvent $A$ and GPL detected by their yellow-gold reaction to orcinol/sulphuric acid reagent.

had a reduced amount of total radioactivity and exhibited a lower percentage of macrophage uptake. Therefore, it was decided that macrophage monolayers would be exposed to $\left[{ }^{3} \mathrm{H}\right] \mathrm{Man}$ radiolabelled GPL and subsequently examined for degradation products without washing the monolayers following the initial pulse, as had been done previously (Hooper et al., 1986). A concentration of $200 \mu \mathrm{g} \mathrm{GPL} \mathrm{ml} \mathrm{g}^{-1}\left(4.6 \times 10^{4}{ }^{3} \mathrm{H}\right.$ c.p.m. per mg GPL) was added to monolayers $\left(3.1 \times 10^{5}\right.$ adherent cells) for time periods of $0,24,48$ and $96 \mathrm{~h}$, after which combined spent medium and macrophages were extracted with chloroform/methanol/water $(8: 4: 3$, by vol.) and radioactivity in upper (methanol/water) phase and lower (chloroform) phase was determined. It was observed that $99 \%(\mathrm{SD}, \pm 0 \cdot 2, n=4)$ of the radioactivity was extractable with chloroform at $0,24,48$ and $96 \mathrm{~h}$. These results are comparable to those obtained with the GPL antigens from serovar 20 in our previous study (Hooper et al., 1986).

Examination by TLC of chloroform-extractable material revealed that all of the serovar 4 GPL were present in the chloroform-extractable material throughout the $96 \mathrm{~h}$ following addition of GPL. Following development of TLC plates in solvent A, GPL were identified by their characteristic colour reaction when sprayed with orcinol/sulphuric acid, and their location with respect to purified GPL standards. Examination of the chloroform-extractable material by TLC in solvent A revealed that radioactivity was localized in an area corresponding to GPL (Fig. 5). This same pattern was observed at $0,24,48$ and $96 \mathrm{~h}$.

\section{DISCUSSION}

Because the GPL antigens may play a role in pathogenesis, they have been the focus of research in this laboratory during the last several years (Hooper et al., 1986; Tereletsky \& Barrow, 1983). Using immunocytochemical and radiolabelling techniques, we have precisely defined the superficial location and distribution of the GPL antigens on the mycobacterial cell wall (Tereletsky \& Barrow, 1983) and demonstrated that these mycobacterial components are relatively inert to macrophage degradation (Hooper et al., 1986). These findings represent the first direct evidence that supports previous suggestions regarding the inertness of the superficial $\mathrm{L}_{1}$ layer (Draper \& Rees, 1970) and indicate that the GPL are important in pathogenesis. In addition, more recent studies in this laboratory have revealed that the GPL, or their metabolites, have the ability to alter lymphocyte function (Brownback \& Barrow, 1988).

In an effort to better examine macrophage degradation of GPL, we have developed procedures for radiolabelling the oligosaccharide portion of these potentially important 
mycobacterial components. The choice of radiolabelled fucose, mannose and methionine to radiolabel the oligosaccharide was based upon their commercial availability and cost effectiveness, and the results of previous studies (Barrow et al., 1980; Ginsberg, 1960; Hooper et al., 1986; Touster, 1962).

Under the conditions of this study, $\left[{ }^{3} \mathrm{H}\right] \mathrm{Fuc}$ gave the least incorporation of radiolabel into native lipids for serovars 4 and 20. Examination of the distribution of radioactivity in the $\left[{ }^{3} \mathrm{H}\right]$ Fuc-labelled lipids from serovars 4 and 20 by TLC revealed that only a minimal amount was incorporated into the GPL. Comparison of these results with previous studies (Hooper et al., 1986) indicated that incorporation of $\left[{ }^{3} \mathrm{H}\right] \mathrm{Fuc}$ was not sufficient for macrophage degradation studies, therefore its use in this investigation was discontinued. Use of $\left[{ }^{3} \mathrm{H}\right] \mathrm{Man}$ and $\left[{ }^{3} \mathrm{H}\right] \mathrm{Met}$ proved to be more favourable for incorporation into native lipid and GPL; of particular importance was the ability of $\left[{ }^{3} \mathrm{H}\right] \mathrm{Man}$ to radiolabel $2-\mathrm{O}-\mathrm{MeFuc}$, which is present in the oligosaccharide determinant of serovars 4 and 20. This latter accomplishment will not only increase our ability to examine degradation of GPL by various enzymic systems, but it will also allow for a more efficient way of exploring the biosynthetic pathway of these mycobacterial lipids.

Based on the present findings, speculations can be made regarding radiolabelling of the oligosaccharide moiety of the GPL from other serovars in the $M$. avium complex. The fact that $\left[{ }^{3} \mathrm{H}\right]$ Met incorporated methyl groups into the methylated sugars of serovars 4 and 20 further substantiates the observation that GPL from other serovars in the $M$. avium complex can be radiolabelled because of the presence of 3,4-di- $O$-MeRha on the fatty acyl-peptide core (Brennan et al., 1981 a, b; Brennan \& Goren, 1979). In addition, serovars such as 8, 9, 16, and 25 can be radiolabelled at sites coinciding with the methylated sugars 3- $O$-methylglucose, 2,3-di- $O$ methylfucose, 4-O-methylrhamnose and 2-O-methylfucose, which have been identified as a part of their oligosaccharide moiety (Brennan et al., $1981 a, b$ ). Likewise, the monosaccharides 2,3-di$O$-MeFuc and 2-O-MeFuc, which are found in the oligosaccharide determinant of serovars 9 and 25 (Brennan et al., 1981 $a, b$ ), should be radiolabelled with $\left[{ }^{3} \mathrm{H}\right] \mathrm{Man}$.

Because $\left[{ }^{3} \mathrm{H}\right] \mathrm{Man}$ allowed for specific incorporation of radiolabel into the oligosaccharide determinant, it was chosen as the radiolabel for subsequent macrophage degradation experiments in an effort to extend our previous studies involving GPL antigens and their possible role in pathogenesis (Hooper et al., 1986). Although use of $\left[{ }^{3} \mathrm{H}\right] \mathrm{Met}$ resulted in a higher degree of radiolabel incorporation into the GPL antigens, it was not specific for the oligosaccharide determinant, but instead resulted in the radiolabelling of 3,4-di- $O$-MeRha, which is the terminal sugar on the fatty acyl-peptide unit $\left(R_{1}\right.$, Fig. 1).

The macrophage pulsing experiments indicate that the maximum level of serovar $4 \mathrm{GPL}$ association with murine peritoneal macrophages takes place somewhere slightly above the 200 $250 \mu \mathrm{g} \mathrm{ml}^{-1}$ level observed for GPL antigens from serovar 20 (Hooper et al., 1986). Although the maximum uptake of serovar $4 \mathrm{GPL}$ was not determined in this study, it was decided to use a concentration of $200 \mu \mathrm{g} \mathrm{ml}^{-1}$ per $3.2 \times 10^{5}$ cells to be consistent with our previous study involving serovar $20 \mathrm{GPL}$ antigens (Hooper et al., 1986). The reason for the low level of serovar 4 GPL association with macrophages was not determined; however, the explanation may have to do with the difference in the GPL structure of the two serovars. From previous studies, it appears that the oligosaccharide determinant of the GPL from serovars 4 and 20 is very similar, differing only in the terminal sugar of the oligosaccharide moiety; serovar 4 contains 4-OMeRha (McNeil et al., 1987), whereas serovar 20 has 2-O-MeRha (Barrow et al., 1980). Although this difference is minor, it may account for the variability in GPL association properties observed in the studies reported here. We have noted in our studies that the GPL from serovar 4 do not disperse as well in the tissue culture medium as do the GPL from serovar 20. This may have contributed to the differences in macrophage association for GPL from serovars 4 and 20.

Because of the reduced amount of radioactivity incorporated with the use of $\left[{ }^{3} \mathrm{H}\right] \mathrm{Man}$ and the reduced percentage uptake of the serovar 4 GPL as compared to that of the serovar 20 antigens (Hooper et al., 1986), the previous experimental design was modified in this investigation. Potential GPL degradation was assessed by examining combined macrophage and spent 
medium pools. Following extraction of lipid from spent medium and macrophage monolayers after exposure to $\left[{ }^{3} \mathrm{H}\right] \mathrm{Man}$-radiolabelled GPL for periods up to $96 \mathrm{~h}$, it was observed $99 \%$ of the radioactivity from the macrophage cultures remained in the chloroform-extractable material, suggesting that little degradation of the GPL of serovar 4 had occurred. Because these results were consistent with previous findings (Hooper et al., 1986) and indicate that little, if any, GPL degradation had occurred, the delineation of antigen products in spent medium and macrophage monolayers was not attempted. These results, in addition to previous findings (Hooper et al., 1986), further substantiate that the GPL are slowly metabolized mycobacterial components and indicate that their accumulation in host tissue may be a real consideration with respect to infections involving members of the $M$. avium complex.

It should be pointed out that these studies are limited by the fact that only resident peritoneal macrophages were utilized in the GPL degradation studies. It is unlikely that the GPL are completely inert to degradation in an intact host system. Indirect evidence for this conclusion is derived from our recent observation that GPL as well as their metabolites may act as immunomodulators (Brownback \& Barrow, 1988). Thus, the unelicited macrophages used in the current and previous (Hooper et al., 1986) study may not have possessed the enzymic capability to degrade the GPL. Now that we have methods for radiolabelling specific sites on the GPL structure, it will be possible to examine whether other macrophage populations or enzymic systems have the capability to degrade these mycobacterial components.

This research was supported by Public Health Service grant AI-21946 of the National Institute of Allergy and Infectious Diseases, by funds from the American Foundation for AIDS Research, and by a TCOM Faculty Research Grant. We thank Paul Brownback and Esther Wright for their technical assistance in the purification of lipid antigens and TLC procedures.

\section{REFERENCES}

BarRow, W. W. \& BrenNan, P. J. (1982). Immunogenicity of type-specific C-mycoside glycopeptidolipids of mycobacteria. Infection and Immunity 36, 678-684.

Barrow, W. W., Ullom, B. P. \& Brennan, P. J. (1980). Peptidoglycolipid nature of the superficial cell wall sheath of smooth-colony-forming mycobacteria. Journal of Bacteriology 144, 814-822.

Brennan, P. J. \& GoRen, M. B. (1979). Structural studies on the type-specific antigens and lipids of the Mycobacterium avium-Mycobacterium intracellulareMycobacterium scrofulaceum serocomplex. Journal of Biological Chemistry 254, 4205-4211.

BrenNaN, P. J., Souhrada, M., Ullom, B., McClatchy, J. K. \& Goren, M. B. (1978). Identification of atypical mycobacteria by thin-layer chromatography of their surface antigens. Journal of Clinical Microbiology 8, 374-379.

Brennan, P. J., Aspinall, G. O. \& Nam Shin, J. E. $(1981 a)$. Structure of the specific oligosaccharides from the glycopeptidolipid antigens of serovars in the Mycobacterium avium-Mycobacterium intracellulare-Mycobacterium scrofulaceum serocomplex. Journal of Biological Chemistry 256, 6817-6822.

Brennan, P. J., Mayer, H., Aspinall, G. O. \& Nam SHIN, J. E. (1981 b). Structures of the glycopeptidolipid antigens from serovars in the Mycobacterium avium/Mycobacterium intracellulare/Mycobacterium scrofulaceum serocomplex. European Journal of Biochemistry 115, 7-15.

Brownback, P. E. \& Barrow, W. W. (1988). Modified lymphocyte response to mitogens after intraperitoneal injection of glycopeptidolipid anti- gens from Mycobacterium avium complex. Infection and Immunity 56, 1044-1050.

Davidson, P. T., Khanijo, V., Goble, M. \& MouldING, T. S. (1981). Treatment of disease due to Mycobacterium intracellulare. Reviews of Infectious Diseases 3, 1052-1059.

Dimitrijevich, S. D., Johnson, M. M. \& Barrow, W. W. (1986). One-step chromatographic procedure for purification of mycobacterial glycopeptidolipid antigens. Journal of Chromatography 377, 345-349.

DRAPER, P. \& ReES, R. J. W. (1970). Electrontransparent zone of mycobacteria may be a defence mechanism. Nature, London 228, 860-861.

Fainstein, V., Bolivar, R., Mavligit, G., Rios, A. \& LUNA, M. (1982). Disseminated infection due to Mycobacterium avium-intracellulare in a homosexual man with Kaposi's sarcoma. Journal of Infectious Diseases 145, 586.

GINSBERG, V. (1960). Formation of guanosine diphosphate L-fucose from guanosine diphosphate Dmannose. Journal of Biological Chemistry 235, 21962201 .

Greene, J. B., Sidhu, G. S., Lewin, S., Levine, J. F., Masur, M., Simberkoff, M. S., Nicholas, P., Good, R. C., Zolla-Pazner, S. B., Pollock, A. A., TAPPER, M. L. \& HolzMan, R. S. (1982). Mycobacterium avium-intracellulare: a cause of disseminated life-threatening infection in homosexuals and drug abusers. Annals of Internal Medicine 97, 539-546.

HOOPER, L. C., Johnson, M. M., KhERA, V. R. \& BARROW, W. W. (1986). Macrophage uptake and retention of radiolabelled glycopeptidolipid antigens associated with the superficial $\mathrm{L}_{1}$ layer of Mycobac- 
terium intracellulare serovar 20. Infection and Immunity 54, 133-141.

Horrocks, R. H. \& Manning, G. B. (1949). Partition chromatography on paper: identification of reducing substances in urine. Lancet 1, 1042-1045.

KieHn, T. E., Edwards, F. F., Brannon, P., Tsang, A. Y., Maio, M., Gold, J. W. M., Whimbey, E., Wong, B., MCClatchy, J. K. \& ARMSTRONG, D. (1985). Infections caused by Mycobacterium avium complex in immunocompromised patients: diagnosis by blood culture and fecal examination, antimicrobial susceptibility tests, and morphological and seroagglutination characteristics. Journal of Clinical Microbiology 21, 168-173.

MASUR, H. (1982). Mycobacterium avium-intracellulare: another scourge for individuals with acquired immunodeficiency syndrome. Journal of the American Medical Association 248, 3013.

McNeil, M., Tsang, A. Y. \& Brennan, P. J. (1987). Structure and antigenicity of the specific oligosaccharide of Mycobacterium avium serotype 4, the dominant mycobacterium isolated from patients with Acquired Immune Deficiency Syndrome. Journal of Biological Chemistry 262, 2630-2635.

RosenZweig, D. Y. (1976). Course and longterm follow-up of 100 cases of pulmonary infection due to M. avium-intracellulare complex. American Review of Respiratory Diseases 113 (Suppl.), 55.

SCHAEFER, W. B. (1965). Serologic identification and classification of the atypical mycobacteria by their agglutination. American Review of Respiratory Diseases 92, 85-93.

TereletsKy, M. J. \& BARRow, W. W. (1983). Postphagocytic detection of glycopeptidolipids associated with the superficial $\mathrm{L}_{1}$ layer of Mycobacterium intracellulare. Infection and Immunity 41, 1312-1321.

TOUSTER, O. (1962). Carbohydrate metabolism. Annual Review of Biochemistry 31, 415-450.

Yeager, H., JR \& Raleigh, J. W. (1973). Pulmonary disease due to Mycobacterium intracellulare. American Review of Respiratory Diseases 108, 547-552. 